\title{
CT measurements of microparts: Numerical uncertainty determination and structural resolution
}

\author{
Matthias Fleßner ${ }^{1}$, Markus Blauhöfer ${ }^{1}$, Eric Helmecke ${ }^{1}$, Andreas Staude ${ }^{2}$, Tino Hausotte ${ }^{1}$ \\ ${ }^{1}$ Institute of Manufacturing Metrology, Friedrich-Alexander-Universität Erlangen-Nürnberg (FAU), \\ Nägelsbachstr. 25, 91052 Erlangen, Germany \\ matthias.flessner@fau.de \\ ${ }^{2}$ BAM Federal Institute for Materials Research and Testing, Unter den Eichen 87, 12205 Berlin,
} Germany

\begin{abstract}
Numerical uncertainty determination for X-ray computed tomography (X-ray CT or CT) measurements is a topic of recent research. For this approach, a realistic model of the real CT system within a simulation tool is prerequisite. Therefore, all relevant properties of the CT system, including all significant error sources, have to be modelled realistically within a Virtual Metrological CT (VMCT).

For dimensional measurements of microparts, the structural resolution has a strong influence on the measurement result. It is mainly induced by the finite size of the X-ray focal spot and the detector unsharpness. These effects superimpose within the 2D projection data. By examining projection data acquired at varying geometrical magnification and varying tube power, it is possible to separate these error sources and adjust the VMCT accordingly.

For a reliable determination of measurement uncertainty, a verification of the VMCT is necessary. As the final measurement results are derived from the extracted surface data, it is favourable to examine characteristics of the surface data instead of characteristics of the projection or volume data. A possible approach is to use an Aperiodic Spatial Frequency Standard (ASFS) to determine the structural resolution of the real and simulated CT system. The ASFS allows comparing the structural resolution for a variety of sensors by investigating the frequency response of the sensor on geometrical structures of different spatial frequency. This is demonstrated on measurements of an ASFS with four different sensors (CT, fringe projection, stylus profilometer, chromatic aberration probe).
\end{abstract}

Key words: dimensional metrology, computed tomography, microparts, numerical uncertainty determination, structural resolution

\section{Introduction}

Dimensional measurement of microparts with complex geometry are becoming increasingly important, for instance in medical and automotive industry. The parts, with sizes ranging from sub-millimetre to some 10 millimetres, frequently require measurements with sub-micrometre accuracy.

For these reasons, the EMRP project 'Multisensor metrology for microparts in innovative industrial products' was started in June 2013. Aim of this three-year Joint Research Project is a significant improvement of state-of-the-art measurement capabilities of multi-sensor coordinate measuring machines for microparts. The project addresses the specific problems related to high accuracy dimensional measurements of small complex features.
Computed Tomography (CT) is a rather young technology in dimensional metrology, but offers some significant advantages like a contactless measurements of inner geometries with a high point density [1]. This renders CT an attractive technology for measurements of microparts. However, measurement uncertainty determination for CT measurements is still a topic of recent research [2-6]. Additionally, it has to be investigated if the structural resolution of the CT system is sufficient to ensure valid measurement results for small geometrical features.

\section{Numerical measurement uncertainty determination}

Guideline VDI/VDE 2630 part 2.1 [7] describes the determination of measurement uncertainty 
for CT in dimensional metrology with use of calibrated workpieces. The main idea behind this approach is that the complex nature of a CT measurement makes it very complicated to determine the impact of every single influence factor on the final measurement result individually. Instead, repeated measurements of a calibrated workpiece are conducted and the measurement uncertainty is derived from a statistical evaluation of the measurement results. In the process, it is assumed that the results of the measurements contain the sum of all influences. To ensure a realistic determination of measurement uncertainty, the conditions during the repeated measurements must comply with the conditions during a real measurement. For instance, the calibrated workpiece must have a similar geometry and material as the actual part, and the acquisition parameters and evaluation strategies must match. Therefore, the measurement uncertainty determined by this method only applies for the specific measurement task investigated. The approach is straightforward, but it is obvious that it is a very time consuming task for costly personnel and equipment.

Instead of carrying out real measurements, an alternative approach is to reproduce these measurements numerically. For this purpose, a simulation tool with a model of the CT system and the measurement process is needed and a number of $2 \mathrm{D}$ projection sets is simulated and processed using the same methods and algorithms as they are used for real measurements. Again, the task specific measurement uncertainty is deduced from a statistical evaluation of the measurement results. To achieve a realistic determination of the measurement uncertainty, all significant error sources of the measurement must be reproduced realistically within the simulation tool.

There have already been some investigations regarding this approach [8, 9], but its validity still has to be proven for CT systems dedicated for dimensional metrology and their superior accuracy compared to conventional CT systems.

Although this approach requires a large amount of computing power and a large effort to model the CT system correctly, its advantages are obvious:

- the task of uncertainty determination is moved away from expensive equipment,

- uncertainty determination for internal and hidden geometries is possible without calibration,
- as only the CAD model of the part is needed, predetermination is possible.

For these reasons, a Virtual Metrological CT (VMCT) is being developed within the 'microparts' project to enable the numerical uncertainty determination for CT measurements of microparts carried out with the CT system of the Institute of Manufacturing Metrology. For this, the simulation software 'aRTist' (analytical Radiographic Testing inspection simulation tool) [10] by BAM is used.

To ensure the validity of the determined measurement uncertainty, a verification of the VMCT is necessary. In the course of this, several properties of the simulated and real measurements are being compared, for instance the signal-to-noise ratio (SNR) or the modulation transfer function (MTF). However, for dimensional measurements, the surface determination and the association of geometrical features (e.g. planes, circles, cylinders, etc.) with the extracted surface points are essential. For this reason, the significance of properties that describe the projection and volume data (like SNR and MTF) is limited. Therefore, a verification of the validity of surface datasets deduced from simulated measurements is preferable.

According to guideline VDI/VDE 2630 part 1.3 [11], the structural resolution is described as 'the size of the smallest structure that can still be measured dimensionally'. For dimensional measurements of microparts, naturally the structural resolution of the CT system has a strong impact on the measurement results. For this reason, this paper will discuss the adjustment of the parameters spot size and detector unsharpness within the VMCT and a novel method for the verification of the VMCT regarding the structural resolution.

\section{Adjustment of simulation}

The unsharpness of the $2 \mathrm{D}$ projection data, which ultimately limits the structural resolution achievable by a CT system, is primarily caused by two characteristics of a CT system: the size of the focal spot of the X-ray tube and the unsharpness of the detector. Especially for high tube power and large geometric magnification, the effect of the finite size of the X-ray spot is dominant [1]. For low geometric magnification, the detector unsharpness (mainly caused by scatter effects within the detector) is dominant [12].

It is not trivial to separate these effects experimentally, as they superimpose within the projection data. Therefore, a simple mathematical model is introduced: the effect of 

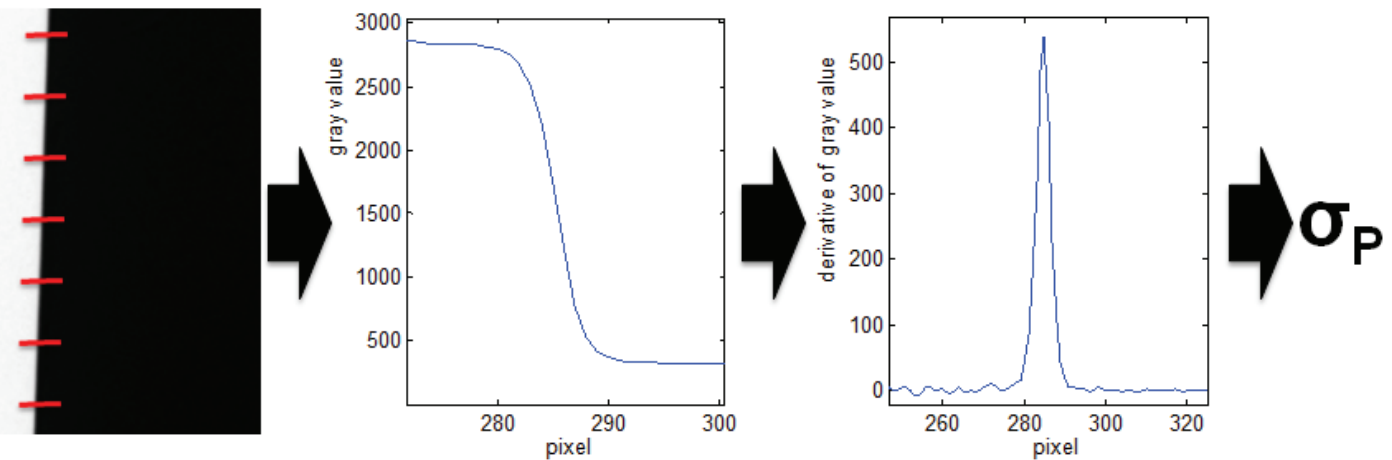

Fig. 1. Evaluation of the unsharpness within the projection data. The derivative of the transition from high to low grey values within the projection data resembles a Gaussian curve. By fitting a Gaussian function to the curve, the parameter $\sigma_{P}$ characterizing the unsharpness of the transition is obtained

the focal spot size is modelled as proportional to the tube power $P$ and, with $M$ being the geometric magnification, proportional to $(M-1)$ (this relation can easily be shown geometrically). The detector unsharpness, however, is modelled as constant, as it is independent from these parameters. Both effects, the focal spot and the detector unsharpness, are modelled as Gaussian functions (with the width of these functions described by the parameters $\sigma_{\text {spot }}$ and $\sigma_{\text {detector }}$ ), while the effect on the projection data is the convolution of these two functions.

By investigating the transition from high to low grey values of the projection image of a sharp edge of a copper plate, it is possible to determine the unsharpness within the 2D projection data (see Fig. 1). With increasing unsharpness, the transition becomes increasingly blurry. As the derivative of this curve resembles a Gaussian curve, a fit of a Gaussian function to the curve is performed. This yields $\sigma_{\mathrm{P}}$, a parameter describing the width of the curve and therefore also describing the unsharpness of the projection data. It can be shown that eq. (1) characterises the relation between the mentioned parameters.

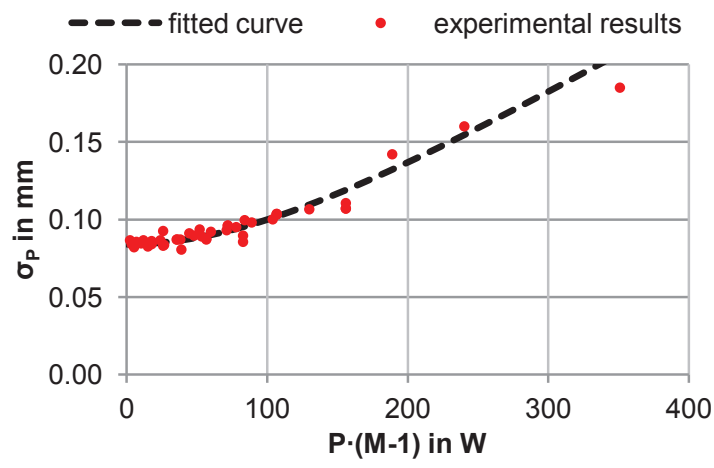

Fig. 2. Experimentally determined values of $\sigma_{P}$ for varying geometrical magnification and tube power. By fitting a theoretical curve according to eq. (1) to the data points, it is possible to separate the effects of spot size and detector unsharpness.

$$
\sigma_{P}=\sqrt{\left(\sigma_{\text {spot }} \cdot P \cdot(M-1)\right)^{2}+\sigma_{\text {detector }}^{2}}
$$

Determining $\sigma_{\mathrm{P}}$ for different tube powers $P$ and magnifications $M$, and fitting a curve according to eq. (1) to the data points makes it possible to calculate values for the parameters $\sigma_{\text {spot }}$ and $\sigma_{\text {detector }}$ (see Fig. 2). For small values of $P \cdot(M-1)$, the focal spot size is considerably smaller than the voxel size and thus the detector unsharpness is dominant. For larger values of $P \cdot(M-1)$, this represents measurements with high magnification and large focal spot size, the impact of the spot size is dominant. This makes it possible to separate the error sources and to adjust the simulation tool according to the parameters calculated by fitting the curve. As a result, for arbitrary magnification and tube power, the projections simulated by the VMCT exhibit the same unsharpness as corresponding real projections.

\section{Investigations of structural resolution for verification of the VCMT}

When it comes to dimensional measurements, the focal spot size and the detector unsharpness directly limit the structural resolution. For this reason, comparing the structural resolution of simulated and real measurements is a solid approach for the verification of the VCMT. However, it is favourable not only to investigate the projection or volume data, but to examine the surface data, as the dimensional measurements are carried out on the calculated surface data.

The MTF is often used for medical CTs to determine the resolution of the system [13]. However, it does only include the volume data and not the extracted surface. Therefore, other approaches that include the surface data are being investigated to determine the structural resolution and to develop an alternative method apart from the method described in the annex of VDI/VDE 2630 part 1.3 [11, 14-19]. 
In this paper, an approach using an Aperiodic Spatial Frequency Standard (ASFS) is discussed. It tries to combine the advantages of the approaches of Arenhart et al. [17, 18] and Illemann et al. [16] and is described in more detail in [19]. The ASFS features small aperiodic, from sinusoidal shapes deviating surface structures. Therefore, performing a Fourier analysis on the surface data yields a wide spectrum of spatial frequencies. By comparing the amplitudes of different spatial frequencies within the surface data of the CT measurement with a high resolution reference measurement, the frequency response (the transmission, or alternatively, damping of the amplitudes) of the CT system depending on the spatial frequency can be determined. From this information, conclusions on the structural resolution of the sensor can be drawn. It has been shown that the ASFS is a promising approach for the determination of the structural resolution of a CT system [19].

A cross section of a simulated measurement of an ASFS is depicted in Fig. 3. Due to different effects (e.g. influence of focal spot size and detector unsharpness, reconstruction, surface determination algorithm), the extracted surface severely differs from the surface of the CAD object that was used as input for the simulation. Edges are rounded, small structures are no longer recognizable and amplitudes of high spatial frequencies are damped.

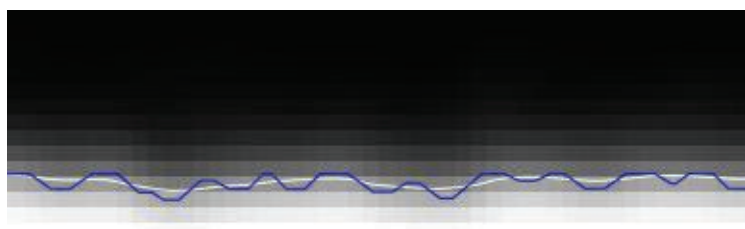

Fig. 3. Cross section of a simulated CT measurement of an ASFS. The extracted surface (white) lacks the high resolution information from the

CAD data (blue) that was used as input for the simulation.

The investigations carried out in [19] show that it is possible to examine the structural resolution of a simulated CT measurement by using the frequency spectrum of the CAD data as reference. To verify the VMCT, additional investigations regarding the structural resolution of real measurements are required. Therefore, a high resolution reference measurement of the ASFS is necessary. In the following, the frequency response for measurements of an ASFS with different sensors will be compared.

\section{Comparison of different sensors}

For the investigations in this paper, a linear ASFS was examined (see Fig. 4). It is made of acrylic glass and was manufactured using a laser cutter. Due to the very low production costs, this standard is suitable for determining the structural resolution of any CT. Compared to the ASFS investigated in [19], a larger variety of sensors is capable of measuring the linear ASFS (as for instance a stylus profilometer for reference measurement). The length of the measured profile is $80 \mathrm{~mm}$, with geometrical structures with a typical size of approx. $400 \mu \mathrm{m}$.

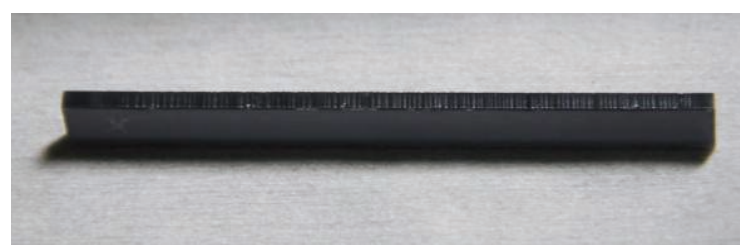

Fig. 4. Photograph of an ASFS.

The profile of the linear ASFS was measured with four different sensors:

- Werth TomoCheck 200 3D with CT sensor, voxel size $44 \mu \mathrm{m}$,

- fringe projection system GOM ATOS Compact Scan 2M, mean point spacing of $21 \mu \mathrm{m}$,

- stylus profilometer Taylor Hobson Form TalySurf Series $2 \mathrm{PGI}$, diamond tip radius of $2 \mu \mathrm{m}$,

- FRT MicroGlider with chromatic aberration probe, measuring spot diameter of $5 \mu \mathrm{m}$.

For the fringe projection system, it has to be noted that it is a challenging measurement task, as the surface is not cooperative for the sensor. The measured amplitudes of the spatial frequencies are depicted in Fig. 5. Using the high resolution stylus profilometer measurement as reference, it is clearly visible that the structural resolution of the fringe projection measurement is worst. With increasing spatial frequency, the measurement process increasingly damps the amplitudes of the surface structures. This effect occurs for rather small spatial frequencies (i.e. large structures). The measurement carried out with the chromatic aberration probe shows a comparable structural resolution as the reference measurement of the stylus profilometer. The structural resolution of the CT measurement is still superior to that of the fringe projection measurement, but for smaller structures (i. e. larger spatial frequency) the amplitudes are moderately damped. 


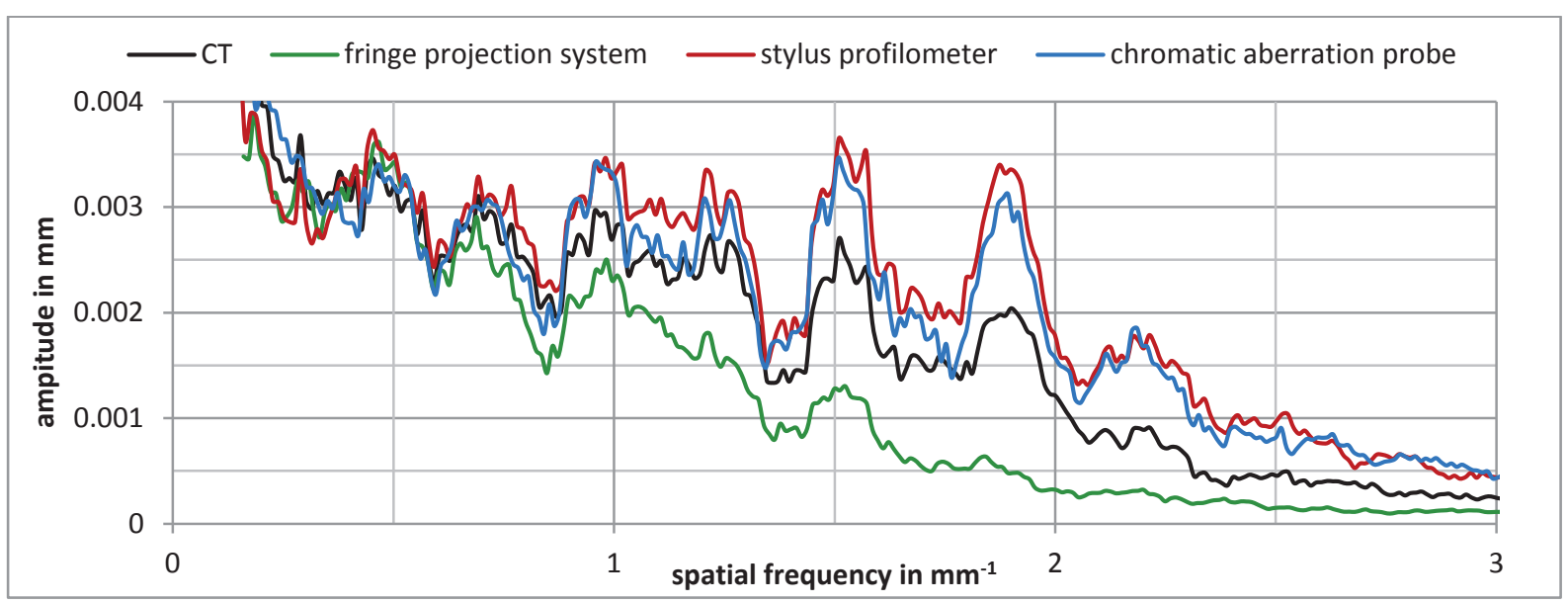

Fig. 5. FFT of measured data for measurements of an ASFS with different sensors.

The investigations suggest that the ASFS is a promising approach to compare the structural resolution of different sensors. The structures investigated experimentally were still rather large, so other manufacturing processes as laser cutting will be taken into consideration for future designs of the ASFS, to allow investigations regarding spatial frequencies relevant for measurements of microparts.

\section{Conclusions}

Using a straightforward approach, the unsharpness within the 2-D projections is determined. By investigating projections acquired at different geometrical magnifications and tube powers, it is possible to separate the effects of the focal spot size and the detector unsharpness and use the results to adjust the simulation software to the characteristics of the real CT system.

For the verification of the VMCT, it is not sufficient to examine characteristics of the projection and volume data, as they do not include the process of surface extraction. Therefore, it is advised to investigate characteristics of the surface dataset, for instance the structural resolution. The presented approach allows examining the damping of different spatial frequencies of different sensors. Therefore, using an ASFS with small surface structures is a possible approach for the verification of the VMCT for measurements of microparts.

\section{Acknowledgement}

The authors would like to thank Andreas Loderer and Heiko Schmidt (Institute of Manufacturing Metrology) for performing the comparison measurements with the fringe projection system and the chromatic aberration probe. The presented research was carried out within the EMRP project 'Multi-sensor metrology for microparts in innovative industrial products'. The EMRP is jointly funded by the EMRP participating countries within EURAMET and the European Union.

\section{References}

[1] J.-P. Kruth, M. Bartscher, S. Carmignato, R. Schmitt, L. De Chiffre, A. Weckenmann, Computed tomography for dimensional metrology, CIRP Annals - Manufacturing Technology 60 (2) (2011) 821-842. doi:10.1016/j.cirp.2011.05.006.

[2] W. Dewulf, K. Kiekens, Y. Tan, F. Welkenhuyzen, J.-P. Kruth, Uncertainty determination and quantification for dimensional measurements with industrial computed tomography, CIRP Annals - Manufacturing Technology 62 (1) (2013) 535-538. doi:10.1016/j.cirp.2013.03.017.

[3] P. Müller, J. Hiller, A. Cantatore, L. De Chiffre, A study on evaluation strategies in dimensional $X$ ray computed tomography by estimation of measurement uncertainties, International Journal of Metrology and Quality Engineering 3 (02) (2012) 107-115. doi:10.1051/ijmqe/2012011.

[4] R. Schmitt, C. Niggemann, Uncertainty in measurement for x-ray-computed tomography using calibrated work pieces, Measurement Science and Technology 21 (5) (2010) 054008. doi:10.1088/0957-0233/21/5/054008.

[5] M. Bartscher, M. Neukamm, U. Hilpert, U. Neuschaefer-Rube, F. Härtig, K. Kniel, K. Ehrig, A. Staude, J. Goebbels, Achieving traceability of industrial computed tomography, Key engineering materials 437 (2010) 79-83.

[6] M. Bartscher, U. Hilpert, D. Fiedler, Ermittlung der Messunsicherheit von Computertomographie-Messungen am Beispiel eines Zylinderkopfs (Determination of the Measurement Uncertainty of Computed Tomography Measurements Using a Cylinder Head as an Example), tm - Technisches 
Messen 75 (3) (2008) 178-186. doi:10.1524/teme.2008.0822.

[7] VDI/VDE 2630 Blatt 2.1: Computed tomography in dimensional metrology - Determination of the uncertainty of measurement and the test process suitability of coordinate measurement systems with CT sensors (draft), Beuth Verlag, Berlin, 2013.

[8] J. Hiller, Abschätzung von Unsicherheiten beim dimensionellen Messen mit industrieller Röntgen-Computertomographie durch Simulation, Der Andere Verlag, 2011.

[9] J. Hiller, L. M. Reindl, A computer simulation platform for the estimation of measurement uncertainties in dimensional X-ray computed tomography, Measurement 45 (8) (2012) 21662182. doi:10.1016/j.measurement.2012.05.030.

[10] C. Bellon, A. Deresch, C. Gollwitzer, G.-R. Jaenisch, Radiographic simulator artist: Version 2, in: Proc. of 18th World Conference on Nondestructive Testing, Durban, South Africa, 2012.

[11] VDI/VDE 2630 Blatt 1.3: Computed tomography in dimensional measurement - Guideline for the application of DIN EN ISO 10360 for coordinate measuring machines with CT-sensors, Beuth Verlag, Berlin, 2011.

[12] U. Zscherpel, K. Osterloh, U. Ewert, Unsharpness characteristics of digital detectors for industrial radiographic imaging, Journal of Nondestructive Testing 9 (5) (2004) 1-8.
[13] W. A. Kalender, Computed tomography: fundamentals, system technology, image quality, applications, John Wiley \& Sons, 2011.

[14] D. Weiß, Q. Shi, C. Kuhn, Measuring the 3D resolution of a micro-focus $\mathrm{X}$-ray CT setup, in: Proceedings of iCT Wels 2012, 2012.

[15] S. Carmignato, A. Pierobon, P. Rampazzo, M. Parisatto, E. Savio, CT for Industrial Metrology - Accuracy and Structural Resolution of CT Dimensional Measurements, in: Proceedings of iCT Wels 2012, 2012.

[16] J. Illemann, M. Bartscher, O. Jusko, F. Härtig, U. Neuschaefer-Rube, K. Wendt, Procedure and reference standard to determine the structural resolution in coordinate metrology, Measurement Science and Technology 25 (6) (2014) 064015. doi:10.1088/09570233/25/6/064015.

[17] F. A. Arenhart, V. C. Nardelli, G. D. Donatelli, M. C. Porath, Investigation of the CT-induced random surface deviations using a multi-wave standard, in: Proceedings of iCT Wels 2012, 2012.

[18] F. A. Arenhart, V. C. Nardelli, G. D. Donatelli, Characterization of the surface content transfer function of CT measuring systems, in: Proceedings of iCT Wels 2014, 2014.

[19] M. Fleßner, N. Vujaklija, E. Helmecke, T. Hausotte, Determination of metrological structural resolution of a CT system using the frequency response on surface structures, in: MacroScale, 2014. 